\title{
Neutrino Non-Standard Interactions: Complementarity between LHC and Oscillation Experiments
}

\author{
K. S. Babu, ${ }^{1}$ Dorival Gonçalves, ${ }^{1}$ Sudip Jana, ${ }^{2}$ and Pedro A. N. Machado ${ }^{3}$ \\ ${ }^{1}$ Department of Physics, Oklahoma State University, Stillwater, OK, 74078, USA \\ ${ }^{2}$ Max-Planck-Institut für Kernphysik, Saupfercheckweg 1, 69117 Heidelberg, Germany \\ ${ }^{3}$ Fermi National Accelerator Laboratory, Batavia, IL, 60510, USA
}

\begin{abstract}
We investigate the complementarity between LHC searches and neutrino experiments in probing neutrino non-standard interactions. Our study spans the theoretical frameworks of effective field theory, simplified model and an illustrative UV completion, highlighting the synergies and distinctive features in all cases. We show that besides constraining the allowed NSI parameter space, the LHC data can break important degeneracies present in oscillation experiments such as DUNE, while the latter play an important role in probing light and weakly coupled physics undetectable at the LHC. This talk is based on results presented in hep-ph 2003.03383 [1].
\end{abstract}

Keywords: NSI, LHC, neutrino, oscillation

DOI: 10.31526/ACP.BSM-2021.28

\section{INTRODUCTION}

In spite of its vast success, the Standard Model (SM) does not shed any light on the origin of neutrino masses. Overwhelming experimental evidence has shown that neutrinos of different flavors oscillate among one another, which cannot occur without small neutrino masses. New physics is necessary for generating such masses, and thus the study of neutrinos offers a promising window for physics beyond the Standard Model.

The new physics associated with neutrino masses can lie in a vast range of energy scales: from sub-GeV to the TeV region, even reaching unification scales of order $10^{14} \mathrm{GeV}$. This poses a phenomenological challenge that should be addressed with multiple experiments probing different energy scales, and in combination with suitable theoretical frameworks. For instance, neutrino Non-Standard Interactions (NSI) with matter can change the neutrino oscillation probabilities [2], where the momentum transfer is negligible, $q^{2} \rightarrow 0$ (for a summary of current status of NSI see Ref. [3]). Therefore, the impact of new physics in oscillation experiments is well described by Effective Field Theory (EFT). In contrast, at high energy colliders, the momentum transfer can be sizable, $q^{2} \sim \mathrm{TeV}^{2}$, possibly leading to direct production of new states, where the consistency of EFT may no longer be guaranteed $[4,5,6,7,8,9]$. Here, a well suited framework should at least include the new degrees of freedom, as in simplified models, or may display an even richer phenomenology, as in a UV complete scenario.

The purpose of this article is to highlight the complementarity of neutrino experiments such as DUNE and collider searches in probing NSIs, across this multitude of frameworks: from the EFT to simplified models, and an illustrative UV completion. In Sec. II we define EFT and simplified scenarios and use them to evaluate the LHC sensitivity to NSIs, in Sec. III we discuss the complementarity between LHC and oscillation physics, in Sec. IV we present an illustrative UV completion, and in Sec. V we conclude.

\section{FROM EFTS TO SIMPLIFIED MODELS}

Neutrino oscillation probabilities are modified in a medium in the presence of NSI, which are generally parametrized in the EFT framework as:

$$
\mathcal{L}_{\mathrm{NSI}}=-2 \sqrt{2} G_{F} \epsilon_{\alpha \beta}^{f Y}\left(\bar{v}^{\alpha} \gamma_{\mu} v^{\beta}\right)\left(\bar{f} \gamma^{\mu} P_{X} f\right)
$$

where $\epsilon_{\alpha \beta}^{f Y}$ defines the strength of the $v-f$ interaction, $\alpha, \beta \in\{e, \mu, \tau\}, f=\{u, d, e\}$, and $X=L(R)$, i.e., $P_{L}\left(P_{R}\right)$ is the left (right) chiral projector.

These new physics contributions can arise from higher dimensional operators that are invariant under the SM gauge symmetry. The dominant effects are expected to come from dimension six operators such as

$$
\frac{1}{\Lambda^{2}}\left(\bar{L}_{\alpha} \gamma_{\mu} L_{\beta}\right)\left(\bar{q} \gamma^{\mu} P_{X} q\right)
$$


where $L$ is the lepton doublet. It follows that $\epsilon_{\alpha \beta}=-1 /\left(2 \sqrt{2} G_{F} \Lambda^{2}\right)$. One would expect prohibitively strong constrains on such operators from charged lepton flavor violation processes [10]. However, flavor diagonal operators can avoid these constraints and lead to observable NSI [11].

Dimension 8 operators of the type

$$
\frac{1}{\Lambda^{4}}\left(\overline{H L}_{\alpha} \gamma_{\mu} H L_{\beta}\right)\left(\bar{q} \gamma^{\mu} P_{X} q\right)
$$

where $H$ is the Higgs doublet [5], do not directly suffer from charged lepton flavor violation constraints, although there are other limits arising from non-unitarity of the PMNS matrix, oblique electroweak corrections, etc. [12]. If the mass of the mediator inducing Eq. (1) is below the electroweak scale, charged lepton flavor violation constraints may even be absent [13, 14].

While for oscillation experiments, we can safely take an agnostic approach to the origin the NSI operators and apply Eq. (1), at the energy scales and couplings probed at the LHC, the validity of the EFT approach is no longer guaranteed. This discussion is similar to recent considerations about the interplay between dark matter (DM) searches at the LHC and low energy direct detection experiments $[15,16,17,18,19,20]$. Namely, while bounds from DM direct detection experiments on new physics can be interpreted in the EFT regime through operators like $\left(\bar{\chi} \gamma_{\mu} \chi\right)\left(\bar{q} \gamma^{\mu} q\right) / \Lambda^{2}$, the same does not hold true at the LHC, where the momentum transfers can go beyond the validity of EFT. Simplified models, in which the force mediator is dealt with explicitly, have been shown to be more appropriate for collider studies. Adopting a simplified model approach, we parametrize the NSI as

$$
\mathcal{L}_{\mathrm{NSI}}^{\mathrm{Simp}}=\left(g_{v}^{\alpha \beta} \bar{v}_{\alpha} \gamma^{\mu} P_{L} v_{\beta}+g_{q i}^{\gamma} \bar{q}_{i} \gamma^{\mu} P_{X} q_{i}\right) Z_{\mu}^{\prime} \text {, }
$$

where $Z_{\mu}^{\prime}$ denotes the new force carrier with arbitrary mass $M_{Z^{\prime}}[4,6]$. Such simplified models have been used in the context of dark matter study, and the parameter space of such models have been constrained by LHC experiments.

Manifestations of dark matter and non-standard neutrino interactions at colliders would look quite similar, both involving large amount of missing transverse energy. A powerful probe of these interactions is the study of mono-jet signatures, in which QCD initial state radiation leads to quark and gluon emission, $p p \rightarrow j+\mathbb{E}_{T}$ with $j=q, \bar{q}, g$. In this context, it has been shown that constraints from LHC $8 \mathrm{TeV}$ data are more stringent for $M_{Z^{\prime}} \gtrsim 100 \mathrm{GeV}$ with the sensitivity reaching $\epsilon \lesssim 10^{-2}[6,7]$.

To estimate the current and high-luminosity LHC sensitivity to NSIs, we will recast the recent jets plus missing energy searches from ATLAS [21] into the simplified model of Eq. (4). The limit $M_{Z^{\prime}} \gg \sqrt{s}$ can be identified as the EFT in Eq. (1). For our analysis, we generate the signal sample $p p \rightarrow v \bar{v} j$ with MADGRAPH5AMC@NLO $[22,23]$, simulating the hadronization and underlying event effects with PYTHIA8 [24]. Detector effects are simulated with the DELPHES3 package [25].

Following the recent $13 \mathrm{TeV}$ ATLAS mono-jet study [21], we define jets with the anti- $k_{t}$ jet algorithm and radius parameter $R=0.4, p_{T j}>30 \mathrm{GeV}$ and $|\eta|<2.8$ via FASTJET [26]. Events with identified electrons with $p_{T}>20 \mathrm{GeV}$ or muons $p_{T}>10 \mathrm{GeV}$ in the final state are vetoed. To suppress the $Z+$ jets and $W+$ jets backgrounds, the events are selected with $E_{T}>250 \mathrm{GeV}$ recoiling against a leading jet with $p_{T j 1}>250 \mathrm{GeV},\left|\eta_{j 1}\right|<2.4$, and azimuthal separation $\Delta \phi\left(j_{1}, \vec{p}_{T, m i s s}\right)>0.4$. Events with more than four jets are vetoed.

Although flavor diagonal NSIs may interfere with the SM background $Z(v \bar{v})+$ jets, we find it to be negligible in the region of interest for the LHC sensitivity. Therefore, the diagonal and non-diagonal NSIs result in equivalent bounds at LHC. Note also that, in fixing the total width, the number of signal events is proportional to $\epsilon^{2} \equiv\left(\sum_{\alpha, \beta}\left|\epsilon_{\alpha \beta}\right|^{2}\right)$ for both on-shell and off-shell production, and thus we use $\epsilon$ to quantify the LHC sensitivity to NSIs. This eases the comparison to neutrino experiments without any further assumptions about the $g_{v}$ vs. $g_{q}$ ratio.

For concreteness, we will assume that neutrino NSIs arise in the simplified model (4) as

$$
\epsilon_{\alpha \beta}^{u}=\epsilon_{\alpha \beta}^{d} \equiv \epsilon_{\alpha \beta}=\frac{\left(g_{v}\right)_{\alpha \beta} g_{u, d}^{V}}{2 \sqrt{2} G_{F} M_{Z^{\prime}}^{2}},
$$

where $g_{u, d}^{V}=g_{u, d}^{L}+g_{u, d}^{R}$ is the quark vector current. We shall assume that axial-vector coupling $g_{q i}^{A}=0$ and generation independent $Z^{\prime}$ interactions with quarks $\left(g_{q}^{V} g_{q i}^{V}, \forall i\right)$ for our numerical results, except in the context of an explicit model where these relations are not realized.

A major limitation of such searches is associated with the overwhelming SM backgrounds, $p p \rightarrow Z(v v) j$ and $p p \rightarrow W(\ell v) j$, which suffer from large theoretical uncertainties, including higher-order QCD and electroweak corrections. Nevertheless, recently efforts have been made to improve the signal sensitivity in both experimental and theoretical fronts [27]. These include further exploration of background control regions and state-of-the-art Monte Carlo simulations, resulting in suppressed background uncertainties and augmented sensitivity to new physics. These advancements lead to suppressed systematic uncertainties that pave the way to stronger NSI constraints at the high-luminosity LHC.

In Fig. 1, we present the LHC sensitivity to NSIs at 95\% CL, estimated for two LHC integrated luminosity scenarios: $36.1 \mathrm{fb}^{-1}$ and $3 \mathrm{ab}^{-1}$. For the lower luminosity case, ATLAS [21] provides a limit on the signal events for ten signal regions. They differ by increasing $\mathbb{E}_{T}$ thresholds. We define the NSI constraint by the most sensitive signal region (black line). For the high-luminosity scenario, we obtain the backgrounds from ATLAS in the same ten $\mathbb{E}_{T}$ thresholds. The NSI sensitivity is obtained from the signal region that maximizes the significance $\mathcal{S}=N_{\mathcal{S}} / \sqrt{N_{b}+\left(\delta \sigma_{b} \cdot N_{b}\right)^{2}}$, where $N_{s}$ and $N_{b}$ are the signal and background events, respectively, and $\delta \sigma_{b}$ is the background uncertainty. To evaluate the impact of systematic uncertainties at the high-luminosity LHC, the sensitivities are evaluated in two scenarios: $\delta \sigma_{b}=1 \%$ and 3\% [27]. The future high-luminosity LHC sensitivity is shown as a blue region, in which the impact of such uncertainties is conveyed as a band. The resulting LHC constraint is maximal for mediators 


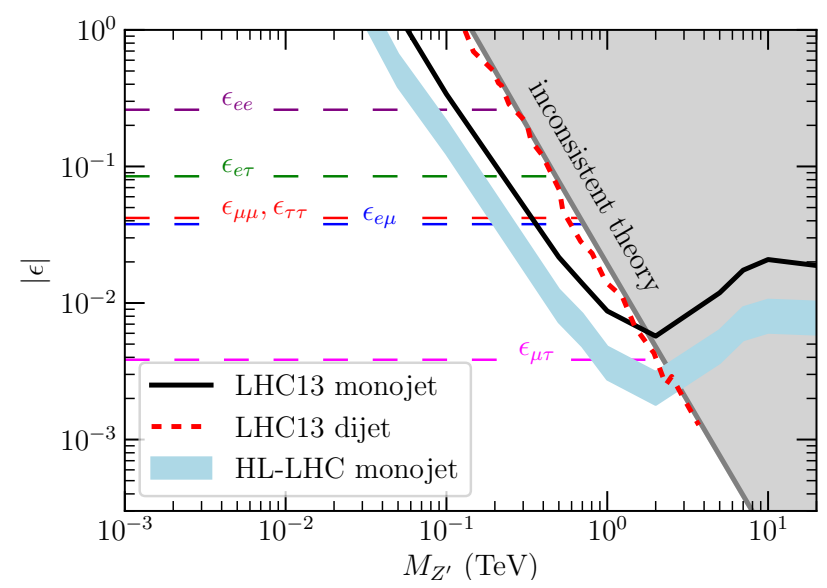

FIGURE 1: Constraints on neutrino non-standard interactions from LHC data and neutrino experiments [28] as a function of the mediator mass $M_{Z^{\prime}}$, assuming $\epsilon_{\alpha \beta} \equiv \epsilon_{\alpha \beta}^{u}=\epsilon_{\alpha \beta}^{d}$. Note that LHC constraints are independent of neutrino flavor. We assumed $\Gamma_{Z^{\prime}} / M_{Z^{\prime}}=0.1$ here.

masses of order $M_{Z^{\prime}} \sim 2 \mathrm{TeV}$, reaching $\epsilon \lesssim 2 \times 10^{-3}$. Flavor dependent bounds on NSI from neutrino experiments [28] are shown as dashed lines and will be discussed in the following section.

In addition to the jets plus missing energy searches, there are other relevant LHC constraints to this simplified model arising from di-jet resonance searches. We use a combination of these limits from ATLAS and CMS [29, 30, 31, 32, 33, 34, 35, 36]. To set the most conservative bound from these data sets, we assume that the coupling of the resonance to neutrinos, $g_{v}$, saturates the chosen width $\Gamma_{Z^{\prime}} / m_{Z^{\prime}}=0.1$. For this choice, the value of $g_{v}$ turns out to be always below 3 . These searches are complementary to mono-jets displaying significant sensitivities at large resonance masses, $m_{Z^{\prime}} \gtrsim 2 \mathrm{TeV}$, see Fig. 1 .

We can identify the EFT regime for the LHC when the mass of the mediator is much above the scale of the process involved. This can clearly be seen in Fig. 1, as the bound on $\epsilon$ does not change for mediator masses above $\sim 5 \mathrm{TeV}$. In addition, a robust argument can be made to estimate the validity of this EFT at the LHC. For any fixed ratio $\Gamma_{Z^{\prime}} / M_{Z^{\prime}}$, we can write the following inequality

$$
|\epsilon| \leq \frac{\sqrt{3} \pi}{\sqrt{N} G_{F} M_{Z^{\prime}}^{2}} \frac{\Gamma_{Z^{\prime}}}{M_{Z^{\prime}}} .
$$

This constraint originates from the fact that the total width of the $Z^{\prime}$ should be larger than the partial widths to $q_{i} \bar{q}_{i}$ and $v \bar{v}$ : $\Gamma_{Z^{\prime}} \geq M_{Z^{\prime}} /(24 \pi)\left(g_{v}^{2}+3 N\left\{\left(g_{u}^{V}\right)^{2}+\left(g_{d}^{V}\right)^{2}\right\}\right)$, where we assumed decay to a single neutrino flavor. $N$ here is the number of quark flavors below the threshold for $Z^{\prime} \rightarrow \bar{f} f$ decay, with each flavor multiplied by a respective phase space factor. The phase space factor for quark flavor $f$ is $P_{f}=\left(1-\frac{2 m_{f}^{2}}{M_{Z^{\prime}}^{2}}\right) \sqrt{1-\frac{4 m_{f}^{2}}{M_{Z^{\prime}}^{2}}}$ (with axial vector coupling set to zero). Considering non-zero axial couplings would make the constraint more stringent. Thus, above $t \bar{t}$ threshold, $N=5+P_{t}$. Assuming $g_{u}^{V}=g_{d}^{V}$ leads to the "inconsistent region" (gray shaded) in Fig. 1. Considering narrower $Z^{\prime}$ makes the constraint stronger, while broader $Z^{\prime}$ implies non-perturbativity $\left(\Gamma_{Z^{\prime}}\right.$ greater than roughly half $\left.M_{Z^{\prime}}\right)$. Therefore, traditional EFT analyses at the LHC using four-fermion operators like Eq. (1) will typically not be valid, at least having simple/minimal UV completions in mind.

\section{COMPLEMENTARITY BETWEEN LHC AND NEUTRINO EXPERIMENTS}

Differently from the LHC, the effects of NSIs in neutrino oscillations strongly depend on the flavor structure of the NSI and the oscillation channel being studied.

In Fig. 1, we show the limits on $\left|\epsilon_{\alpha \beta}\right|$ for each flavor combination, derived from the global fit [28] using neutrino oscillation and COHERENT data (see also Ref. [37]), where all other NSI parameters were marginalized over. We have combined the limits on $\epsilon_{\alpha \beta}^{u}$ and $\epsilon_{\alpha \beta}^{d}$ at 95\% CL in [28] in quadrature, $\sigma_{\alpha \beta}=\left[\left(\sigma_{\alpha \beta}^{u}\right)^{-2}+\left(\sigma_{\alpha \beta}^{d}\right)^{-2}\right]^{-1 / 2}$. These constraints on NSI parameters span two orders of magnitude, showing the strong dependency on the flavor structure of the NSI.

The flavor dependence of NSIs on neutrino oscillations goes beyond different sensitivities. The effects of different NSIs and/or variations of the standard oscillation parameters can, in some cases, compensate each other and lead to well known degeneracies. Disentangling those is a difficult task at neutrino facilities. In contrast, the mono-jet signal at the LHC, $p p \rightarrow \bar{v}_{\alpha} v_{\beta} j$, does not distinguish between different choices of $(\alpha, \beta)$; i.e., they all lead to the same observables. Besides constraining the currently allowed NSI parameter space, this feature can be further exploited to break relevant degeneracies.

To make this point manifest, we present two examples of degeneracy breaking in the following. In Fig. 2, we show the 95\% CL bounds on $\left|\epsilon_{e e}\right|$ vs. $\left|\epsilon_{e \tau}\right|$ from Ref. [38], obtained by combining current oscillation and scattering data with future DUNE sensitivity. 


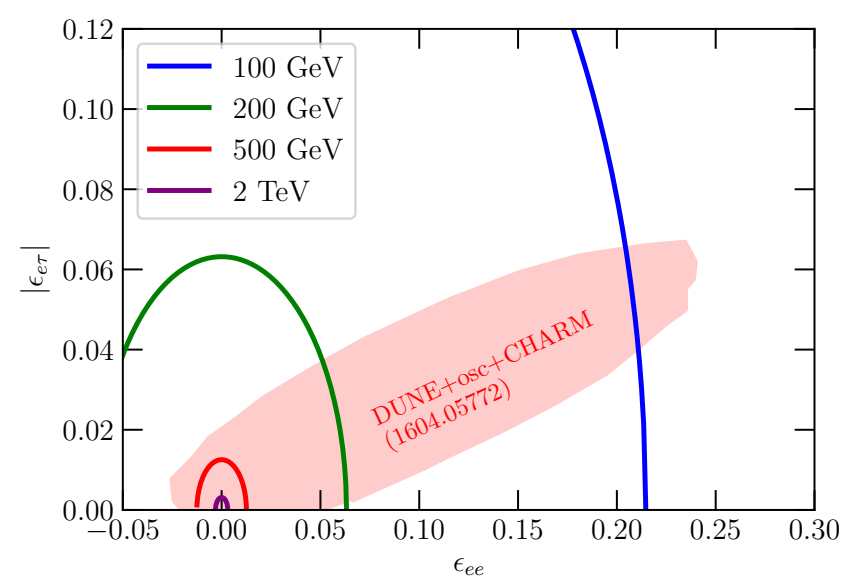

FIGURE 2: Constraints on neutrino non-standard interactions from LHC data, for different mediator masses as labeled, with $\Gamma_{Z^{\prime}} / M_{Z^{\prime}}=0.1$, assuming $\epsilon_{\alpha \beta} \equiv \epsilon_{\alpha \beta}^{u}=\epsilon_{\alpha \beta}^{d}$. A fit to simulated data for DUNE from Ref . [38] is also included (pink shaded region).

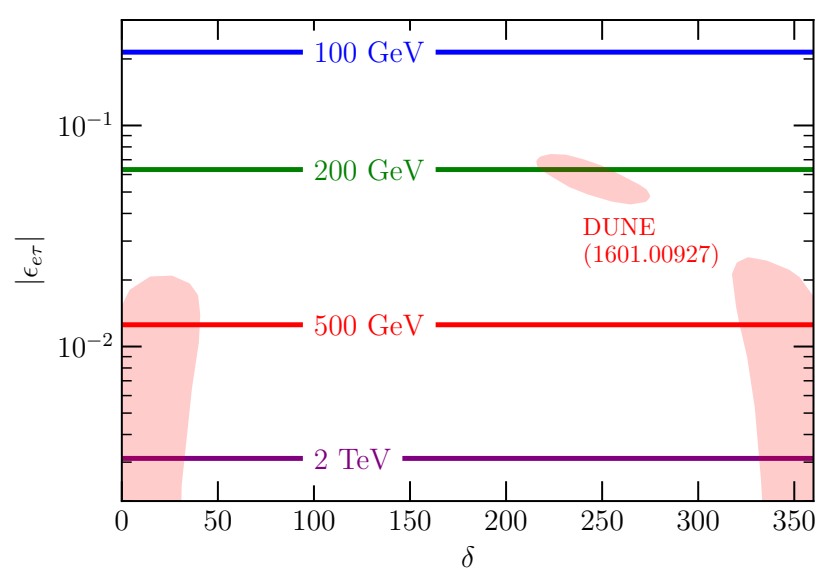

FIGURE 3: An example of degeneracy in the $\left(\epsilon_{e \tau}, \delta\right)$ plane, where $\delta$ is the standard $C P$ violating phase and $\epsilon_{e \tau} \equiv \epsilon_{\mathcal{e} \tau}^{u}=\epsilon_{\mathcal{e} \tau}^{d}$, from future DUNE data taken from Ref. [39] (pink shaded regions are allowed, assuming $\epsilon_{\mathfrak{e} \tau}=\delta=0$ as null hypothesis). We overlay the LHC sensitivity to this NSI parameter for several mediator masses, as labeled, assuming $\Gamma_{Z^{\prime}} / M_{Z^{\prime}}=0.1$.

In Fig. 3, we show the 95\% CL bounds on $\epsilon_{e \tau}$ vs. the usual CP violating phase $\delta$ for the future DUNE experiment from Ref. [39]. Here, $v_{\mu} \rightarrow v_{e}$ and $\bar{v}_{\mu} \rightarrow \bar{v}_{e}$ oscillation channels, driven by the smallest mixing parameter, $\sin ^{2} 2 \theta_{13}$, are crucial to constrain these parameters. These oscillations are significantly affected by matter effects, and channels like $v_{\mu} \rightarrow v_{\tau}, v_{e} \rightarrow v_{\mu}$ are much harder to study due to experimental limitations. The allowed regions from neutrino experiments are shown in pink shaded, while the LHC bound depends on the mediator mass and is depicted as colored lines, as indicated in the figures. It should be noted that, even with the future DUNE experiment, several degeneracies will remain unsolved by oscillation measurements, but could in principle be unravelled by LHC data.

Therefore, several important complementary aspects between LHC and neutrino experiments can already be identified. The LHC sensitivity displays a strong dependence on the mediator mass, but it is free of parameter degeneracies. Neutrino oscillation measurements, on the other hand, exhibit the opposite behavior: significant degeneracies and no mediator mass dependence.

On top of that, there is another complementary aspect that cannot be seen from the figures presented so far. The matter potential induced when neutrinos travel through a medium is not affected by a diagonal, universal contribution (as this just induces an overall phase shift on the neutrino state). On the other hand, LHC data is sensitive to each and all NSI parameters independently. Note also that neutrino oscillations are not sensitive to axial interactions, while LHC data is sensitive to both vector and axial new physics contributions. All these features show the synergies between oscillation measurements and collider data on probing new physics in the neutrino sector. 


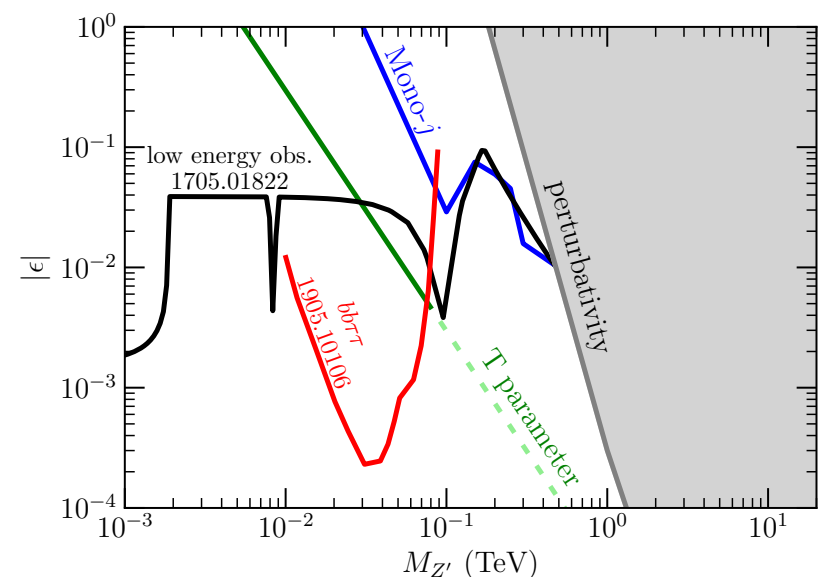

FIGURE 4: Sensitivities to a specific UV complete model realization of non-standard interactions where the $B-L$ number of the third family is gauged [13]. This includes current low energy observables (black), electroweak $T$ parameter (green solid and dotted, see text), future high-luminosity LHC searches for $b b \tau \tau$ final states (red, adapted from Ref. [41]), and LHC mono-jet searches (blue).

\section{TOWARDS A UV COMPLETE SCENARIO}

Any UV complete model of neutrino NSI is expected to provide a more extensive phenomenology, especially since neutrinos are in the same $S U(2)_{L}$ doublet as charged leptons. This further enhances the synergies between LHC and oscillation experiment, as we shall demonstrate in an illustrative UV completion. We show in Fig. 4 the constraints on the UV complete model of Ref. [13] (see Ref. [40] for other constructions with leptonic signals). In this UV completion the $B-L$ number is gauged, but only for the third family. This leads to a new gauge boson that couples more strongly with the third family fermions and results in nonzero $\epsilon_{\tau \tau}$. In Ref. [13] the entire model was specified, and an extensive list of constraints were derived from low energy observables such as neutrino oscillation, $D-\bar{D}$ mixing, $Y$ and $B$ decays, atomic parity violation, and others.

Assuming $\tan \beta=0.5$ (see Ref. [13]), we compare these constraints (black line) with a dedicated $b b \tau \tau$ search [41] (red line), electroweak precision $T$ parameter [42] (green solid and dotted line) and the high-luminosity LHC jets plus missing energy constraint of Sec. II (blue line). The latter displays a distinctive profile due to enhanced light flavors initial state contributions for $M_{Z^{\prime}} \sim M_{Z}$ and $b$-initiated at $M_{Z^{\prime}} \gg M_{Z}$. If $M_{Z}<M_{Z^{\prime}}$, the contribution to the $T$ parameter can in principle be compensated by those stemming from the scalar sector, making this constraint model dependent (indicated by the dotted green line). Finally, we also show the region in which the theory becomes non-perturbative, corresponding to the new gauge coupling being larger than 2.

Low energy constraints, dedicated LHC searches, and missing energy signatures provide strong constraints for different masses of the mediator. For masses below about $10 \mathrm{GeV}$, low energy observables tend to dominate. In the intermediate regime $10-100 \mathrm{GeV}$, dedicated searches for visible signatures at the LHC become more relevant. Finally, from $0.1-1$ TeV LHC mono-jet searches, low energy observables and electroweak precision observables (up to the $T$ parameter model dependence) play the leading role. This makes manifest the complementarities among collider data, oscillation measurements, and other low energy observables.

In this article, we have mainly discussed $Z^{\prime}$ mediated NSI contributions. However, two of the authors in collaboration with B. Dev and A. Thapa have recently presented another comprehensive analysis [11] of neutrino non-standard interactions (NSI) generated by new scalars in radiative neutrino mass models. A new nomenclature has been adopted to classify radiative neutrino mass models, viz., the class of models with at least one SM particle in the loop are dubbed as type-I radiative models, whereas those models with no SM particles in the loop are called type-II radiative models. From NSI perspective, the type-I radiative models are most interesting, as the neutrino couples to a SM fermion (matter field) and a new scalar directly, thus generating NSI at tree-level, unlike type-II radiative models. After taking into account various theoretical and experimental constraints, the maximum possible NSI in all the type-I radiative models has been summarized [11]. In these scenarios also, a powerful probe of these interactions (via $t$ - channel scalar leptoquark exchange) can be the study of mono-jet signatures at the LHC, in which QCD initial state radiation leads to quark and gluon emission, $p p \rightarrow j+E_{T}$ with $j=q, \bar{q}, g$. In case of leptophilic charged scalars, it will lead to mono-photon signature at the lepton collider $e^{+} e^{-} \rightarrow \gamma+\mathbb{E}_{T}$. In Ref. [43], it has been also shown that the light charged scalars in radiative models could give rise to a Glashow-like resonance feature in the UHE neutrino event spectrum at the IceCube neutrino observatory and its high-energy upgrade IceCube-Gen2, which can probe a sizable fraction of the allowed NSI parameter space. While the analysis $[11,43]$ is focused on Zee model, it essentially covers various other theoretically motivated models [44, 45, 46, 47, 48, 49, 50, 51] with light charged scalars.

\section{CONCLUSION}

We have explored the complementarity between neutrino experiments and LHC searches in probing neutrino non-standard interactions. Our analysis covers the full span of theory frameworks: effective field theories, simplified models, and an illustrative UV 
completion. We have shown that the present and high-luminosity LHC sensitivities to NSIs display relevant synergies to oscillation results. Namely, $i$ ) the breakdown of degeneracies among NSI and oscillation parameters, and $i i$ ) sensitivity to new phenomenological signatures at the LHC. As a by-product of our analysis, we have shown that the use of EFT at the LHC in estimating sensitivity to NSIs is not generally theoretically consistent.

\section{References}

[1] K. S. Babu, D. Gonçalves, S. Jana and P. A. N. Machado, Phys. Lett. B 815, 136131 (2021) doi:10.1016/j.physletb.2021.136131 [arXiv:2003.03383 [hep-ph]].

[2] L. Wolfenstein, Phys. Rev. D 17, 2369-2374 (1978) doi:10.1103/PhysRevD.17.2369

[3] P. S. Bhupal Dev, K. S. Babu, P. B. Denton, P. A. N. Machado, C. A. Argüelles, J. L. Barrow, S. S. Chatterjee, M. C. Chen, A. de Gouvêa and B. Dutta, et al. SciPost Phys. Proc. 2, 001 (2019) doi:10.21468/SciPostPhysProc.2.001 [arXiv:1907.00991 [hep-ph]].

[4] S. Davidson and V. Sanz, Phys. Rev. D 84, 113011 (2011) doi:10.1103/PhysRevD.84.113011 [arXiv:1108.5320 [hep-ph]].

[5] Z. Berezhiani and A. Rossi, Phys. Lett. B 535, 207-218 (2002) doi:10.1016/S0370-2693(02)01767-7 [arXiv:hep-ph/0111137 [hep-ph]].

[6] A. Friedland, M. L. Graesser, I. M. Shoemaker and L. Vecchi, Phys. Lett. B 714, 267-275 (2012) doi:10.1016/j.physletb.2012.06.078 [arXiv:1111.5331 [hep-ph]].

[7] D. Buarque Franzosi, M. T. Frandsen and I. M. Shoemaker, Phys. Rev. D 93, no.9, 095001 (2016) doi:10.1103/PhysRevD.93.095001 [arXiv:1507.07574 [hep-ph]].

[8] D. Choudhury, K. Ghosh and S. Niyogi, Phys. Lett. B 784, 248-254 (2018) doi:10.1016/j.physletb.2018.07.053 [arXiv:1801.01513 [hep-ph]].

[9] I. Bischer and W. Rodejohann, Nucl. Phys. B 947, 114746 (2019) doi:10.1016/j.nuclphysb.2019.114746 [arXiv:1905.08699 [hep-ph]].

[10] S. Bergmann and Y. Grossman, Phys. Rev. D 59, 093005 (1999) doi:10.1103/PhysRevD.59.093005 [arXiv:hep-ph/9809524 [hep-ph]].

[11] K. S. Babu, P. S. B. Dev, S. Jana and A. Thapa, JHEP 03, 006 (2020) doi:10.1007/JHEP03(2020)006 [arXiv:1907.09498 [hep-ph]].

[12] M. B. Gavela, D. Hernandez, T. Ota and W. Winter, Phys. Rev. D 79, 013007 (2009) doi:10.1103/PhysRevD.79.013007 [arXiv:0809.3451 [hep-ph]].

[13] K. S. Babu, A. Friedland, P. A. N. Machado and I. Mocioiu, JHEP 12, 096 (2017) doi:10.1007/JHEP12(2017)096 [arXiv:1705.01822 [hep-ph]].

[14] Y. Farzan and I. M. Shoemaker, JHEP 07, 033 (2016) doi:10.1007/JHEP07(2016)033 [arXiv:1512.09147 [hep-ph]].

[15] P. J. Fox, R. Harnik, J. Kopp and Y. Tsai, Phys. Rev. D 85, 056011 (2012) doi:10.1103/PhysRevD.85.056011 [arXiv:1109.4398 [hep-ph]].

[16] G. Busoni, A. De Simone, E. Morgante and A. Riotto, Phys. Lett. B 728, 412-421 (2014) doi:10.1016/j.physletb.2013.11.069 [arXiv:1307.2253 [hep-ph]].

[17] O. Buchmueller, M. J. Dolan and C. McCabe, JHEP 01, 025 (2014) doi:10.1007/JHEP01(2014)025 [arXiv:1308.6799 [hep-ph]].

[18] D. Goncalves, P. A. N. Machado and J. M. No, Phys. Rev. D 95, no.5, 055027 (2017) doi:10.1103/PhysRevD.95.055027 [arXiv:1611.04593 [hep$\mathrm{ph}]]$.

[19] N. F. Bell, G. Busoni and I. W. Sanderson, JCAP 03, 015 (2017) doi:10.1088/1475-7516/2017/03/015 [arXiv:1612.03475 [hep-ph]].

[20] T. Alanne and F. Goertz, Eur. Phys. J. C 80, no.5, 446 (2020) doi:10.1140/epjc/s10052-020-7999-2 [arXiv:1712.07626 [hep-ph]].

[21] M. Aaboud et al. [ATLAS], JHEP 01, 126 (2018) doi:10.1007/JHEP01(2018)126 [arXiv:1711.03301 [hep-ex]].

[22] J. Alwall, M. Herquet, F. Maltoni, O. Mattelaer and T. Stelzer, JHEP 06, 128 (2011) doi:10.1007/JHEP06(2011)128 [arXiv:1106.0522 [hep-ph]].

[23] J. Alwall, R. Frederix, S. Frixione, V. Hirschi, F. Maltoni, O. Mattelaer, H. S. Shao, T. Stelzer, P. Torrielli and M. Zaro, JHEP 07, 079 (2014) doi:10.1007/JHEP07(2014)079 [arXiv:1405.0301 [hep-ph]].

[24] T. Sjostrand, S. Mrenna and P. Z. Skands, Comput. Phys. Commun. 178, 852-867 (2008) doi:10.1016/j.cpc.2008.01.036 [arXiv:0710.3820 [hep$\mathrm{ph}]]$.

[25] J. de Favereau et al. [DELPHES 3], JHEP 02, 057 (2014) doi:10.1007/JHEP02(2014)057 [arXiv:1307.6346 [hep-ex]].

[26] M. Cacciari, G. P. Salam and G. Soyez, Eur. Phys. J. C 72, 1896 (2012) doi:10.1140/epjc/s10052-012-1896-2 [arXiv:1111.6097 [hep-ph]].

[27] J. M. Lindert, S. Pozzorini, R. Boughezal, J. M. Campbell, A. Denner, S. Dittmaier, A. Gehrmann-De Ridder, T. Gehrmann, N. Glover and A. Huss, et al. Eur. Phys. J. C 77, no.12, 829 (2017) doi:10.1140/epjc/s10052-017-5389-1 [arXiv:1705.04664 [hep-ph]].

[28] P. Coloma, I. Esteban, M. C. Gonzalez-Garcia and M. Maltoni, JHEP 02, 023 (2020) doi:10.1007/JHEP02(2020)023 [arXiv:1911.09109 [hep-ph]].

[29] A. M. Sirunyan et al. [CMS], Eur. Phys. J. C 78, no.9, 789 (2018) doi:10.1140/epjc/s10052-018-6242-x [arXiv:1803.08030 [hep-ex]].

[30] A. M. Sirunyan et al. [CMS], JHEP 08, 130 (2018) doi:10.1007/JHEP08(2018)130 [arXiv:1806.00843 [hep-ex]].

[31] [CMS], CMS-PAS-EXO-19-012.

[32] V. Khachatryan et al. [CMS], Phys. Rev. Lett. 117, no.3, 031802 (2016) doi:10.1103/PhysRevLett.117.031802 [arXiv:1604.08907 [hep-ex]].

[33] A. M. Sirunyan et al. [CMS], Phys. Rev. Lett. 120, no.20, 201801 (2018) doi:10.1103/PhysRevLett.120.201801 [arXiv:1802.06149 [hep-ex]].

[34] [CMS], CMS-PAS-EXO-18-012.

[35] A. M. Sirunyan et al. [CMS], Phys. Rev. Lett. 123, no.23, 231803 (2019) doi:10.1103/PhysRevLett.123.231803 [arXiv:1905.10331 [hep-ex]].

[36] A. M. Sirunyan et al. [CMS], JHEP 04, 031 (2019) doi:10.1007/JHEP04(2019)031 [arXiv:1810.05905 [hep-ex]].

[37] B. Dutta, R. F. Lang, S. Liao, S. Sinha, L. Strigari and A. Thompson, JHEP 09, 106 (2020) doi:10.1007/JHEP09(2020)106 [arXiv:2002.03066 [hep$\mathrm{ph}]$.

[38] P. Coloma and T. Schwetz, Phys. Rev. D 94, no.5, 055005 (2016) [erratum: Phys. Rev. D 95, no.7, 079903 (2017)] doi:10.1103/PhysRevD.94.055005 [arXiv:1604.05772 [hep-ph]].

[39] J. Liao, D. Marfatia and K. Whisnant, Phys. Rev. D 93, no.9, 093016 (2016) doi:10.1103/PhysRevD.93.093016 [arXiv:1601.00927 [hep-ph]].

[40] T. Han, J. Liao, H. Liu and D. Marfatia, JHEP 11, 028 (2019) doi:10.1007/JHEP11(2019)028 [arXiv:1910.03272 [hep-ph]].

[41] F. Elahi and A. Martin, Phys. Rev. D 100, no.3, 035016 (2019) doi:10.1103/PhysRevD.100.035016 [arXiv:1905.10106 [hep-ph]].

[42] M. Tanabashi et al. [Particle Data Group], Phys. Rev. D 98, no.3, 030001 (2018) doi:10.1103/PhysRevD.98.030001

[43] K. S. Babu, P. S. Dev, S. Jana and Y. Sui, Phys. Rev. Lett. 124, no.4, 041805 (2020) doi:10.1103/PhysRevLett.124.041805 [arXiv:1908.02779 [hep$\mathrm{ph}]$.

[44] K. S. Babu, S. Jana and M. Lindner, JHEP 10, 040 (2020) doi:10.1007/JHEP10(2020)040 [arXiv:2007.04291 [hep-ph]].

[45] L. Graf, S. Jana, M. Lindner, W. Rodejohann and X. J. Xu, Phys. Rev. D 103, no.5, 055007 (2021) doi:10.1103/PhysRevD.103.055007 [arXiv:2010.15109 [hep-ph]].

[46] A. Bally, S. Jana and A. Trautner, Phys. Rev. Lett. 125, no.16, 161802 (2020) doi:10.1103/PhysRevLett.125.161802 [arXiv:2006.11919 [hep-ph]].

[47] M. Berbig, S. Jana and A. Trautner, Phys. Rev. D 102, no.11, 115008 (2020) doi:10.1103/PhysRevD.102.115008 [arXiv:2004.13039 [hep-ph]].

[48] S. Jana, V. P. K. and S. Saad, Phys. Rev. D 101, no.11, 115037 (2020) doi:10.1103/PhysRevD.101.115037 [arXiv:2003.03386 [hep-ph]].

[49] K. S. Babu and S. Jana, JHEP 02, 193 (2019) doi:10.1007/JHEP02(2019)193 [arXiv:1812.11943 [hep-ph]]. 
[50] E. Bertuzzo, S. Jana, P. A. N. Machado and R. Zukanovich Funchal, Phys. Lett. B 791, 210-214 (2019) doi:10.1016/j.physletb.2019.02.023 [arXiv:1808.02500 [hep-ph]].

[51] E. Bertuzzo, S. Jana, P. A. N. Machado and R. Zukanovich Funchal, Phys. Rev. Lett. 121, no.24, 241801 (2018) doi:10.1103/PhysRevLett.121.241801 [arXiv:1807.09877 [hep-ph]]. 